\title{
Nannizziopsis Immune Reconstitution Inflammatory Syndrome in a Patient with HIV: First Reported Case
}

\author{
Yi Zhao ${ }^{1}$, Mikhail Nozdrin ${ }^{1}$, Alessia Dalla Pria ${ }^{2,3}$, Margherita Bracchi ${ }^{3}$ \\ ${ }^{1}$ Imperial College London School of Medicine, London, UK \\ ${ }^{2}$ Department of Infectious Diseases, Imperial College London, London, UK \\ ${ }^{3}$ Department of HIV Medicine, Chelsea and Westminster Hospital, London, UK
}

Received: $21 / 10 / 2021$

Accepted: $30 / 10 / 2021$

Published: $23 / 11 / 2021$

How to cite this article: Zhao Y, Nozdrin M, Dalla Pria A, Bracchi M. Nannizziopsis immune reconstituition inflammatory syndrome in a patient with HIV: first reported case. EJCRIM 2021;8: doi:10.12890/2021_003021.

Conflicts of Interests: The authors declare there are no competing interests.

This article is licensed under a Commons Attribution Non-Commercial 4.0 License

\section{ABSTRACT}

We describe the case of a 35-year-old HIV-positive male of African origin diagnosed with neurotoxoplasmosis and a Nannizziopsis spp. cavitating pulmonary lesion unmasking immune reconstitution inflammatory syndrome (IRIS).

The patient presented with headache, left hemiparesis and confusion. MRI of the brain showed two space-occupying lesions in the right basal ganglia and left parietal lobe typical for neurotoxoplasmosis. The patient tested positive for HIV and had advanced CD4 lymphopenia. After commencement of antiretroviral treatment, a CT scan of the chest showed a cavitating lesion in the right upper lobe. The diagnosis of Nannizziopsis spp. fungal infection was confirmed by DNA sequencing on a bronchial wash sample. The patient achieved complete recovery with antiretroviral therapy, standard neurotoxoplasmosis treatment and antifungal treatment with voriconazole for 12 weeks.

\section{LEARNING POINTS}

- $\quad$ AIDS patients at risk of multiple concomitant opportunistic infections present a significant diagnostic challenge.

- Unusual pathologies should also be considered in addition to the most common opportunistic pathogens, especially in the context of immune reconstitution inflammatory syndrome.

\section{KEYWORDS}

HIV, immune reconstitution inflammatory syndrome, IRIS, Nannizziopsis

\section{CASE DESCRIPTION}

A 35-year-old man originally from Togo, who had been living in the UK for 2 years, presented with a 2-week history of headache and behavioural change. On admission, he was afebrile, his vital signs were within normal ranges, and the general examination was unremarkable. The neurological examination revealed mild confusion and mild left hemiparesis. A CT of the head showed a right basal ganglia mass lesion with multiloculated peripheral wall post-contrast enhancement, significant mass effect, midline shift and hydrocephalus.

An MRI of the brain (Fig. 1) confirmed the presence of a peripherally enhancing central necrotic lobulated mass lesion. An HIV test performed at admission came back positive. The T lymphocyte CD4+ count was 4 cells $/ \mathrm{mm}^{3}$ (1.4\%) and the HIV viral load was 45,700 copies/ml. A lumbar puncture was not performed as it was deemed too risky (due to mass effect of the lesions) and after confirmation of a negative serum cryptococcus antigen test, antiretroviral therapy with raltegravir and tenofovir-DF/emtricitabine was started. A full-body CT scan was also performed and showed splenomegaly, with no significant lymphadenopathies. 


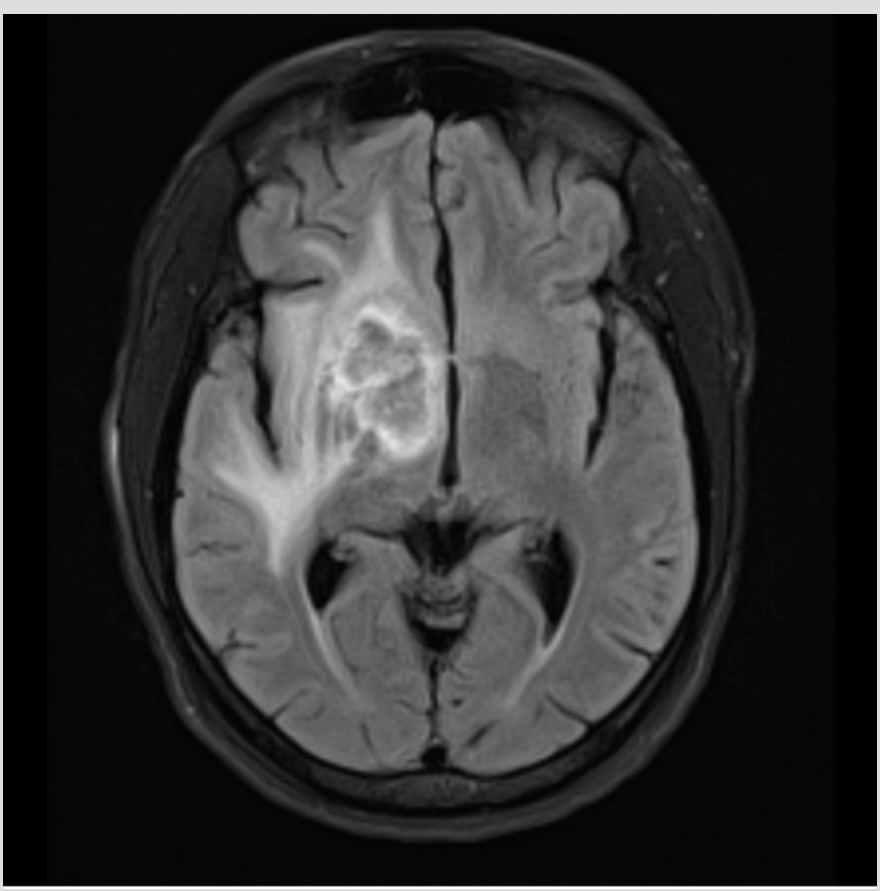

Figure 1. T2-weighted MRI with contrast showing the lesion in the right globus pallidus with extensive white matter signal abnormality in the basal ganglia, most of the right temporal, frontal and parietal lobes, and tracking inferiorly to involve the midbrain and superior pons

A presumptive diagnosis of neurotoxoplasmosis was made also considering positive Toxoplasma serology, and treatment with sulfadiazine, pyrimethamine and folic acid was started. The patient also underwent a full body 18F-FDG PET/CT scan to further evaluate the brain lesions, which showed decreased metabolic activity in comparison with the contralateral hemisphere. Subsequent chest imaging performed after a new onset of fevers showed a new right upper lobe cavitating lesion (15 mm in diameter) (Fig. 2). Bronchoscopy with bronchoalveolar lavage allowed the isolation of filamentous fungi, subsequently identified as Nannizziopsis spp. via DNA sequencing. The minimal inhibitory concentrations (MIC) for amphotericin, itraconazole and voriconazole were $0.5,0.5$ and $0.06 \mu \mathrm{g} / \mathrm{ml}$, respectively. The lesion was thought to be secondary to an unmasking immune reconstitution inflammatory syndrome (IRIS) phenomenon, after 3 weeks of effective antiretroviral therapy with achievement of undetectable viral load and a small improvement in CD4+ T cell count (Fig. 3).

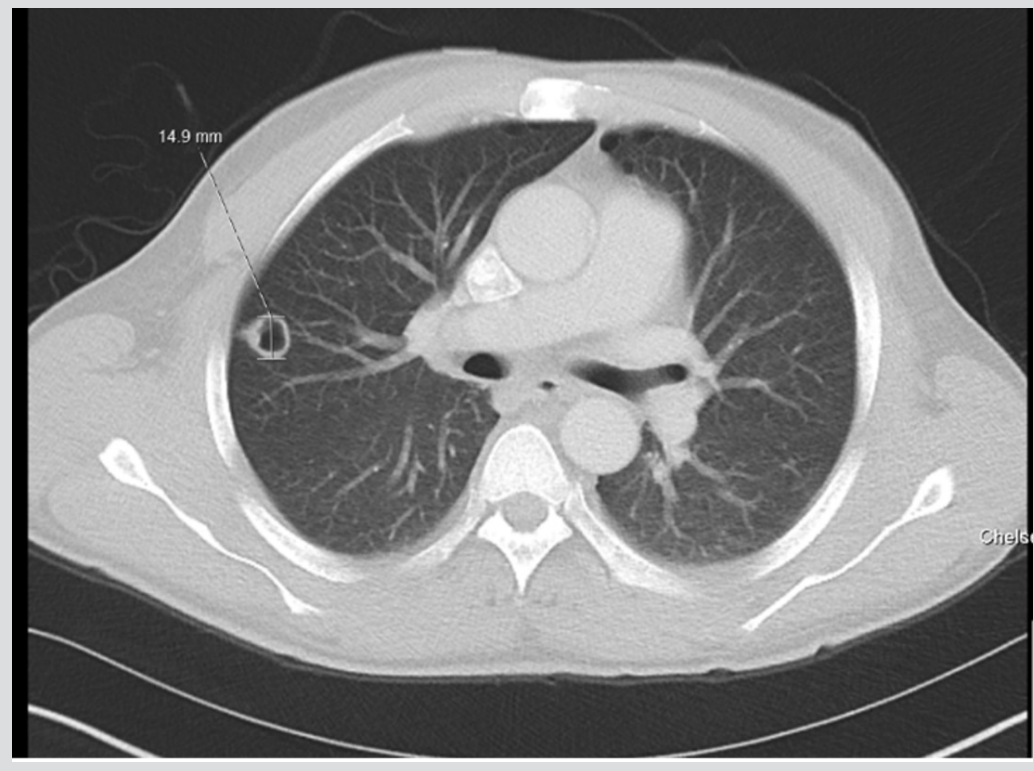

Figure 2. CT of the chest showing a new thick-walled cavitating $15 \mathrm{~mm}$ lesion in the peripheral right upper lobe 
CD4+ Lymphocyte absolute count and HIV viral Load trends

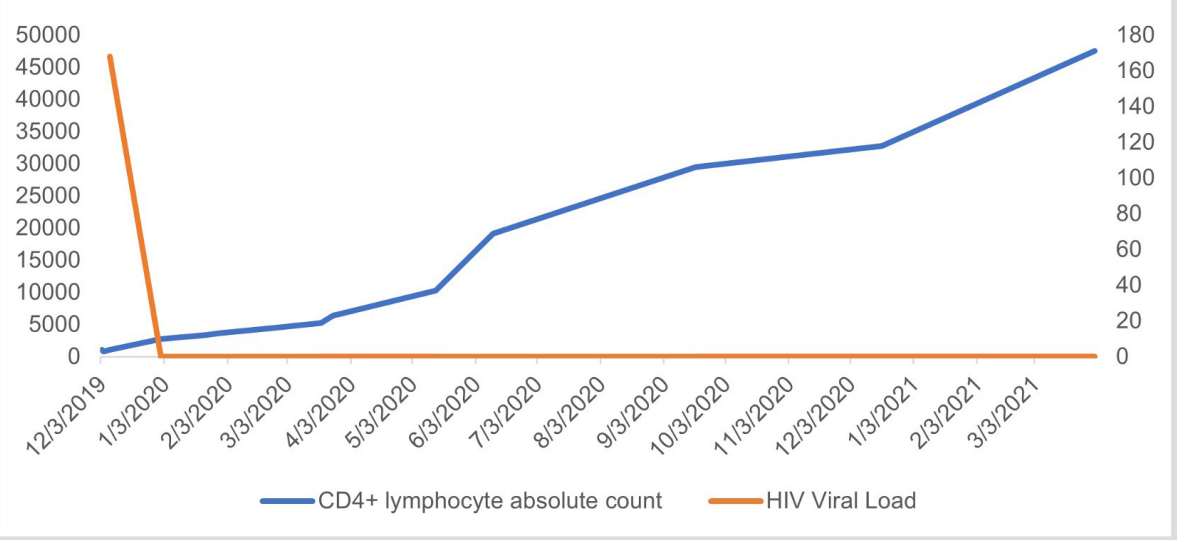

Figure 3. CD4 lymphocyte absolute count (cells/ $\mu$ l) and HIV viral load ( $\mathrm{cp} / \mathrm{ml}$ ) over time. The axis for HIV viral load is on the left, while the axis for CD4 lymphocyte absolute count is on the right

Voriconazole treatment at a dose of $400 \mathrm{mg}$ twice daily on day 1 followed by $200 \mathrm{mg}$ twice daily for 12 weeks achieved good clinical response. The blood $\beta$-D-glucan (BDG) level was used as a marker for treatment. The patient's BDG values during the course of treatment are shown in Fig. 4.

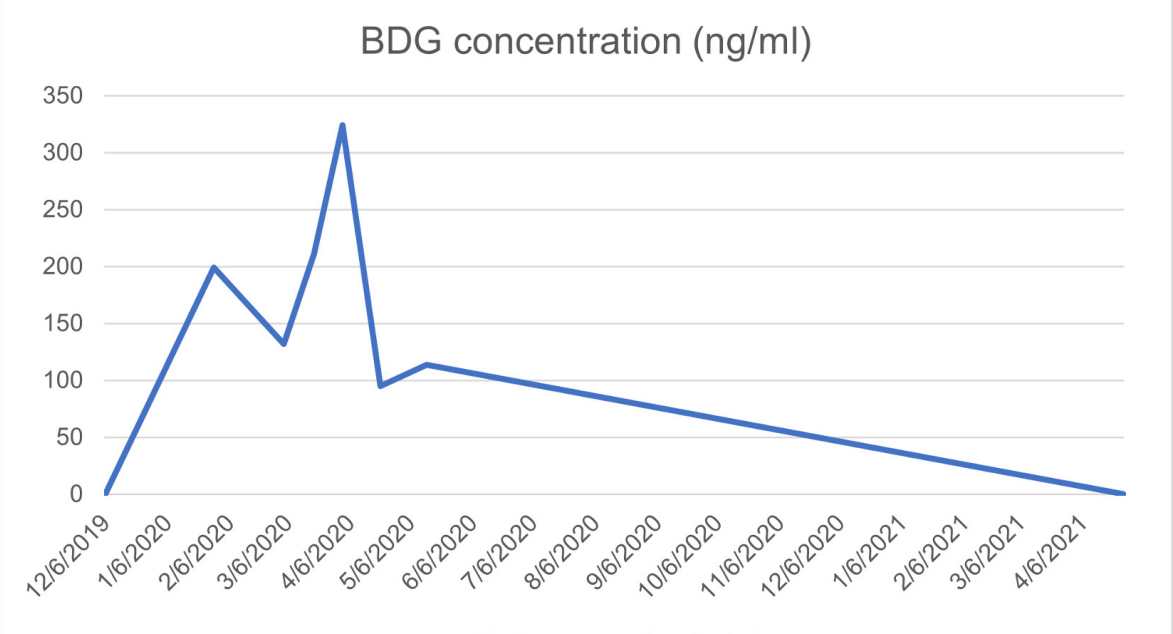

Figure 4. Serum $\beta$-D-glucan (BDG) over time

A repeat CT of the chest after the end of treatment (week 12) showed reduced size of the lesion with resolution of the cavitation. Continuous improvement of the cerebral lesions at repeated brain MRI scans was observed.

At 18-month follow-up, the patient is alive, asymptomatic, and has no neurological sequelae or respiratory symptoms. He is compliant with antiretrovirals with well-controlled HIV (HIV RNA <20 cp/ml; CD4 cell count 171/mm³, 8\%) and negative BDG levels.

A lumbar puncture was performed at resolution of the cerebral oedema 3 months into HIV treatment. Cerebrospinal fluid (CSF) showed only mildly raised proteins $(0.83 \mathrm{~g} / \mathrm{l})$ with normal cell count and negative fungal or bacterial culture. Results were negative for Toxoplasma DNA, fungal culture, BDG and 16S/18S ribosomal RNA (rRNA) gene polymerase chain reaction.

\section{DISCUSSION}

The first documented case of Nannizziopsis infection in humans was in 1982 when a 24-year-old African man presented with osteomyelitis ${ }^{[1]}$. More cases in organ transplant hosts have been subsequently described in the literature ${ }^{[2,3]}$. Nannizziopsis spp. infection remains extremely rare and there is a lack of guidance on optimal management. We report the second case of Nannizziopsis spp. infection in the UK, in a patient with newly diagnosed advanced HIV and concomitant neurotoxoplasmosis. 
Most patients with Nannizziopsis spp. present with HIV infection or other causes of immunodeficiency such as solid organ transplants or haematological malignancies ${ }^{[1-3]}$. Nannizziopsis spp. shares infective features with other dermatophytes which may cause aggressive skin disease ${ }^{[2]}$. The severe form of dermatophytosis is seen in late solid organ transplant recipients ${ }^{[1,3,4]}$. Our patient did not have any skin or nail lesions but had lung involvement, which was previously described in other cases ${ }^{[3]}$. Brain abscesses due to Nannizziopsis have also been reported in patients with advanced HIV infection; in our case, the brain lesions are thought to be more likely cerebral toxoplasmosis, as initial improvement was noticed prior to initiation of antifungal treatment. Moreover, we did not find evidence of fungal infection on CSF (negative culture, BDG and $18 \mathrm{~S}$ ribosomal RNA (rRNA) gene polymerase chain reaction) ${ }^{[1,3]}$.

Cases reported in the literature have been treated with posaconazole, voriconazole, amphotericin B or itraconazole. We choose voriconazole due to previous successful treatment in patients with brain abscesses ${ }^{[1,2]}$, the favourable MIC, the availability of an oral option ${ }^{[5]}$, as well as the absence of clinically significant drug interactions with the patient's antiretroviral and toxoplasma treatment.

Nannizziopsis spp. infections are rare and cases reported in the literature have had variable outcomes. It has been suggested that a decrease in immunosuppressive medications may contribute to a better prognosis ${ }^{[3]}$, especially in patients who have recently had a renal transplant. Patients mostly remained well after treatment was completed with no relapse ${ }^{[1,3]}$.

There have been four reported deaths in patients infected with Nannizziopsis spp. ${ }^{[1-3]}$. Three of four patients were investigated for a different origin of infection, which caused a delay in isolating the relevant strain of Nannizziopsis. For the patient in this case report, Nannizziopsis was identified relatively quickly and appropriate treatment was commenced early. This suggests early identification of the organism and early antimicrobial treatment is important.

Although uncommon, it is important to include rarer co-infections such as Nannizziopsis in the differential diagnosis of severely immunocompromised hosts (e.g., patients with advanced HIV) presenting with cerebral, cutaneous and pulmonary lesions. Unmasking IRIS is a well-described phenomena in severely immunocompromised patients starting on antiretroviral therapy. To our knowledge, this is the first described case of Nannizziopsis pulmonary cavitating lesions presenting as unmasking IRIS.

\section{REFERENCES}

1. Nourrisson C, Vidal-Roux M, Cayot S, Jacomet C, Bothorel C, Ledoux-Pilon A, et al. Invasive infections caused by Nannizziopsis spp. molds in immunocompromised patients. Emerg Infect Dis 2018;24(3):549-552.

2. Baggott A, McGann H, Barton R, Ratner J. Disseminated Nannizziopsis obscura infection in a renal transplant patient- the first reported case. Med Mycol Case Rep 2017;17:2024.

3. Garcia-Hermoso D, Hamane S, Fekkar A, Jabet A, Denis B, Siguier M, et al. Invasive infections with Nannizziopsis obscura species complex in 9 patients from West Africa, France, 2004-2020. Emerg Infect Dis 2020;26(9).

4. Rouzaud, C, Chosidow, O, Brocard, A, Fraitag S, Scemla A, Anglicheau D, et al. Severe dermatophytosis in solid organ transplant recipients: a French retrospective series and literature review. Transplant Infect Dis 2018;20(1). doi: 10.1111/tid.12799.

5. Tang L, Yang X-F, Qiao M, Zhang L, Tang X-W, Qiu H-Y, et al. Posaconazole vs. voriconazole in the prevention of invasive fungal diseases in patients with haematological malignancies: a retrospective study. J Mycol Med 2018;28(2):379-383. 